\title{
El intercambio obras digitales en la nube: la responsabilidad de las empresas tecnológicas por infracciones al copyright
}

\section{Mariliana Rico Carrillo"*}

\begin{abstract}
RESUMEN
El presente trabajo aborda el tratamiento jurídico del intercambio de archivos que contienen obras protegidas por el copyrigth en la nube en la legislación y la jurisprudencia de los Estados Unidos, con la finalidad de determinar si existe responsabilidad de los proveedores de estos servicios por las infracciones cometidas por sus usuarios, y si la normativa actual es idónea para enfrentar la protección del copyright en este entorno. A efectos de cumplir este objetivo, se estudian los principios de la Digital Millenium Copyright Act y la forma en que los jueces han determinado la responsabilidad de estos sujetos en situaciones similares. La investigación finaliza con un análisis de la sentencia del caso Capitol Records vs. MP3tunes donde se trata de manera especifica la responsabilidad de los proveedores de servicios de cloud computing en el intercambio de material protegido por el copyright.
\end{abstract}

\section{PALABRAS CLAVE}

Computación en la nube. Proveedores de servicios en Internet. Propiedad Intelectual. Responsabilidad.

\begin{abstract}
The following article looks into the legal treatment of the exchange of files protected by copyright protected materials, in the cloud in legislation and in the laws of the United States, with the objective to determine if there is responsibility of those who provide the services due to the infringements committed by their users, and if the current regulations are suitable to face the protection of copyright materials in this environment. In order to achieve this objective, we study the principles of the Digital Millennium Copyright Act and the way in which judges have determined the responsibility of these subjects in similar situations. The research ends with an analysis of the ruling of the Capitol Records vs. MP3tunes where the responsibility of providers of cloud computing services in the exchange of material protected by copyright is specifically addressed.
\end{abstract}

\section{KEYWORDS}

Cloud Computing. Internet service providers, Intellectual property, Responsibility

\footnotetext{
*Artículo recibido el 14 de agosto de 2017 y aceptado el 16 de octubre de 2017

*Universidad Católica del Táchira. (mariliana@ricocarrillo.com)
} 
SUM ARIO

1. Introducción

2. El desarrollo tecnológico y la protección de los derechos de autor

3. La protección de la propiedad intelectual en internet

4. La responsabilidad de las empresas que facilitan el acceso a internet

5. Supuestos de responsabilidad

6. La doctrina de la responsabilidad secundaria

7. Los principios de safe harbor y fair use

8. El caso Capitol Records, Inc. v. MP3tunes

9. Conclusiones

\section{Introducción}

En 2011, Equipment Manufacturers International (EMI) Incorporated y catorce compañías discográficas y editoras de música (incluyendo Capitol Records) demandaron ante la Corte de Distrito Sur de Nueva York de los Estados Unidos la responsabilidad de la empresa tecnológica MP3tunes, por suministrar un sistema de almacenamiento en la nube que permitía a sus usuarios guardar e intercambiar obras musicales digitales sujetas a protección bajo la normativa que rige el copyright en este país. ${ }^{1}$ Este caso es conocido como Capitol Records, Inc. v. MP3tunes, LLc. y constituye el núcleo central del presente trabajo, orientado a analizar la responsabilidad que debe atribuirse a las empresas tecnológicas (en particular a los proveedores de servicios en la nube) por proveer una tecnología que permite vulnerar los derechos de autor de obras digitales.

Aunque el modus operandi en este tipo de vulneraciones al copyright ha determinado que los usuarios son los infractores, más que la empresa que ofrece la infraestructura tecnológica. Sin embargo, emI optó por demandar la responsabilidad MP3tunes, alegando que la empresa había contribuido a la infracción al proporcionar los medios que permitían a los usuarios finales violar el copyright de EMI y que además estaba en conocimiento de esta situación. Como punto previo, es importante advertir que si bien es cierto que son los usuarios quienes, aprovechando las funcionalidades de la tecnología, intercambian los archivos digitales y facilitan al público el acceso y uso de obras protegidas sin la correspondiente autorización de sus autores, la configuración

\footnotetext{
1 Véase "Capitol Records, Inc. v. uc, Exp. No. 07-9931 (S.D.N.Y. Agos 13, 2009)", Casetext. [Consulta: 20 de enero, 2017]. Disponible en: https://casetext.com/case/capitol-records-inc-v-mp3tunes-3
} 
de los servicios de internet y la protección de la privacidad de los usuarios dificultan la identificación de los responsables primarios. Esta es la razón por la cual en diferentes oportunidades los afectados han optado por exigir la responsabilidad de las empresas que facilitan dicha tecnología.

Estos hechos, así como la posible contribución y el conocimiento de las empresas tecnológicas de las infracciones cometidas por los usuarios, hacen que sea necesario determinar si existe algún tipo de responsabilidad. De ser así, habría que determinar el tipo de responsabilidad que debe atribuirse a una determinada empresa. En Capitol Records, Inc. v. MP3tunes, LLc, la corte determinó que de acuerdo con los principios de la legislación y jurisprudencia de los Estados Unidos, MP3Tunes era responsable en cierta medida de la vulneración al copyrigth.

La sentencia es clara. Aunque la demandada podía beneficiarse de los principios que permiten la exoneración de responsabilidad en estos casos (básicamente el safe harbor), no cumplió con su obligación de remover las canciones y el material fonográfico que infringía el copyright de los archivos privados de los usuarios; por lo tanto, era responsable de tal infracción. La importancia de esta sentencia es determinante para empresas como Google, Apple y Amazon, que también ofrecen servicios en la nube. Además, ha sido considerada como una victoria para la música administrada desde los sistemas de "nube", pues estableció un determinado ámbito de legalidad al cloud computing. ${ }^{2}$

\section{El desarrollo tecnológico y la protección de los derechos de autor}

El desarrollo tecnológico ha influido decisivamente en la protección de los derechos de autor de diferentes maneras. Uno de los casos más conocidos relacionados con este asunto data de los años ochenta y tuvo lugar en los Estados Unidos. Nos referimos al caso Sony Corp. of America v. Universal City Studio, también conocido como el "caso Betamax". Esta fue la primera vez que la Corte Suprema de los Estados Unidos analizó la responsabilidad de una empresa que introdujo una nueva tecnología que facilitaba la vulneración del copyright.

En este caso, la Corte determinó que, aunque algunos consumidores utilizaban esta tecnología con fines ilícitos, al grabar y comercializar obras protegidas por el copyright, otros aprovechaban sus funcionalidades para grabar la programación televisiva, y ver sus programas favoritos en otro momento. En

\footnotetext{
${ }^{2}$ Aristizabal Velásquez, David, "Luces y sombras de las nuevas tendencias de la regulación de contenidos informáticos en los Estados Unidos de Norteamérica", Revista Ces Derecho, vol. 3, núm. 1, p. 75.
} 
atención a este uso, la grabación personal fue declarada como una exención al copyright bajo la doctrina del fair use (uso legítimo o permitido). En esta oportunidad, la Corte declaró que la venta de equipos que permiten realizar copias, al igual que la venta de otros artículos de comercio, no constituye una infracción contributiva (contributory infringement) si el producto se puede utilizar ampliamente con fines legitimos significativos.

La doctrina Sony se basa en el fair use del material protegido por el copyright y se considera la decisión más importante para equilibrar los intereses de los derechos de los autores con los de las empresas desarrolladoras de tecnología y sus respectivos usuarios. En este caso, la Corte Suprema adoptó una solución que sopesaba el valor y la legitimidad de la nueva tecnología contra el posible daño a los intereses de los titulares del copyright. ${ }^{3}$ Bajo esta doctrina, en un reclamo de infracción del derecho de autor por contributory infringement, basta demostrar que la tecnología puede usarse con una finalidad lícita. ${ }^{4}$ La consecuencia jurídica más importante de la decisión de la Corte fue el establecimiento de una doctrina general para determinar si la creación de un dispositivo o la introducción de una tecnología que permite la copia o la grabación de una obra viola la protección del copyright. En la actualidad la doctrina Sony se utiliza para analizar la responsabilidad de las empresas que ofrecen servicios en internet (en particular las que facilitan el uso de tecnologías que permiten el intercambio de archivos de obras protegidas) a fin de determinar su posible responsabilidad por las infracciones al copyright cometidas por los usuarios. ${ }^{5}$

\section{La protección de la propiedad intelectual en internet}

El acceso gratuito a internet crea la idea del libre acceso a la cultura y a los contenidos digitales en general, sin tomar en cuenta la protección de los derechos de autor. Actualmente, los sistemas de comunicación P2P, las redes sociales, los servicios de cloud computing y otras aplicaciones informáticas permiten reproducir, compartir y almacenar la información. Esto facilita aún más la vulneración a los derechos de propiedad intelectual en este ámbito.

A los efectos de establecer las reglas para lograr el balance entre la protección de los autores y el derecho al acceso a la cultura a través de internet, la Organización Mundial de la Propiedad Intelectual (oMPI) aprobó en 1996 los

\footnotetext{
3 Leary, BRIAN, "Safe Harbor Startups: Liability Rulemaking under the DMCA", New York University Law Review, núm. 87.

4 Antequera Parilli, Ricardo, Estudios sobre derechos de autor y derechos afines. Madrid, Fundación Aisge, 2007, p. 367.

${ }^{5}$ Leary, BRIAN, "Safe Harbor Startups: Liability Rulemaking under the DMcA", New York University Law Review, núm. 87.
} 
conocidos tratados de internet. El propósito es actualizar los tratados internacionales previos sobre derechos de autor a la era digital y proporcionar la protección adecuada a los titulares de derechos de autor derivados de los avances tecnológicos.

El contenido de estos tratados ha sido incorporado en el ordenamiento jurídico de los países que forman parte de la Unión Europea, en los Estados Unidos, así como en los diversos países de la comunidad internacional por conducto de distintos instrumentos normativos. En el caso Europa, la adopción de los principios sobre la protección de la propiedad intelectual en internet reflejados en los tratados de la ompI se llevó a cabo mediante la aprobación de la Directiva 2001/29/CE del Parlamento Europeo y del Consejo, de 22 de mayo de 2001, relativa a la armonización de determinados aspectos de los derechos de autor y derechos afines a los derechos de autor en la Sociedad de la Información. ${ }^{6}$ El texto ha sido incorporado a la legislación interna de los distintos países miembro de la Unión Europea.

En Estados Unidos, fue la Digital Millennium Copyright Act (DMCA), sancionada por el Congreso durante el año 2000, la norma jurídica que se encargó de incorporar los principios de los tratados de internet a la legislación de este país. ${ }^{7}$

\section{La responsabilidad de las empresas que facilitan el acceso a internet}

Las empresas tecnológicas que suministran acceso y servicios en internet se conocen genéricamente por las siglas osP (Online Service Provider). Dentro de estas encontramos a los ISP (Internet Service Provider). La DMCA delimita esta categoría a quienes ofrecen la transmisión, enrutamiento o conexión para comunicaciones en línea a elección del usuario, sin modificación del contenido del material enviado o recibido. En esta definición entra la figura del proveedor de servicios de cloud computing. Éste pone a disposición una plataforma que permite almacenar información digital a discreción de los usuarios del servicio y que puede compartirse fácilmente con otras personas.

\footnotetext{
${ }^{6}$ Esta directiva se encuentra actualmente en proceso de revisión a efectos de adaptar su contenido a los nuevos desarrollos tecnológicos. En el marco de la estrategia de la Unión Europea para la creación de un mercado único digital, durante el año 2016 se publicó en el Diario Oficial la Propuesta de Directiva del Parlamento Europeo y del Consejo sobre el derecho de autor en el mercado único digital.

7 En Estados Unidos, el copyright está protegido por la Copyright Act de 1976, incluida en el Título 17 del U.S. Code. Luego de la aprobación de los tratados de internet, la Copyright Act fue modificada mediante la incorporación de las normas de la DMCA al referido texto legal.
} 
Siguiendo los lineamientos de los tratados de internet, en particular del Tratado de la oMPI sobre Derecho de Autor (TODA), tanto la DMCA como la directiva europea establecen las condiciones para determinar la responsabilidad y las causas de exoneración aplicables a los ISP. Principalmente se basan en la función (la actividad) que desarrolla la empresa tecnológica que presta el servicio en la red y en la conducta de los usuarios.

En cuanto a las limitaciones y exenciones de responsabilidad que amparan a este tipo de prestadores de servicios en el ámbito del copyright, destaca la introducción de los principios de safe harbor, que permiten proteger a los ISP de la responsabilidad por las infracciones a los derechos de autor basadas en ciertas prácticas comunes de la industria. Con el establecimiento de los principios de safe harbor, la DMCA busca equilibrar los intereses de los titulares del copyright y los de las empresas que suministran acceso y alojamiento en la red. Busca, asimismo, la cooperación, minimizar las vulneraciones a los derechos de los autores y proporcionar un mayor grado de certeza a las empresas tecnológicas sobre su responsabilidad por la actuación de los usuarios finales de la tecnología. ${ }^{8}$

El fair use se refiere al uso lícito de la obra. Se basa en el propósito y el carácter del uso e incluye la reproducción en copias o fonogramas para propósitos tales como críticas, comentarios, reportajes y enseñanza. Para determinar el fair use, se deben tener en cuenta diversos factores que deben considerarse caso por caso al momento de analizar si el uso del material protegido sin autorización de su autor es lícito. La incorporación del fair use en la DMCA es acorde con las previsiones del artículo 10 del ToDA. Este dispone que la legislaciones nacionales podrán establecer limitaciones o excepciones en ciertos casos especiales, siempre que no atenten contra la explotación normal de la obra, ni causen perjuicio injustificado a los intereses legítimos del autor. ${ }^{9}$ Los tribunales

\footnotetext{
${ }^{8}$ En el caso Capitol Records, Inc. V., LLC, se pone de manifiesto este propósito: "The DMCA seeks to balance the interests of copyright owners and online service providers by promoting cooperation, minimizing copyright infringement, and providing a higher degree of certainty to service providers on the question of copyright infringement". "Capitol Records, Inc. v. ucc, Exp. No. 07-9931 (S.D.N.Y. Agos 13, 2009)", Casetext. [Consulta: 20 de enero, 2017]. Disponible en https://casetext.com/case/capitol-records-inc-v-mp3tunes-3

${ }^{9}$ Esta disposición incorpora la regla de los "tres pasos", que exige el cumplimiento de los siguientes requisitos para que la exoneración proceda: a) que se trate de supuestos excepcionales (interpretados en forma restrictiva); b) que no atenten contra la explotación moral de la obra; y c) que no causen perjuicio injustificado a los legítimos intereses del autor. Estas excepciones tienen que ver con los denominados usos honrados previstos en el Convenio de Berna para la protección de obras literarias y artísticas. Sobre la aplicación de esta regla como límite a la aplicación de las excepciones legales, véase Carbauo CAScón, FERnANDO, "La propiedad intelectual como objeto del comercio electrónico", en Maria Jesús Moro Almaraz, Autores, consumidores y comercio electrónico, Madrid, Colex, 2004, pp. 68 y 430; GarRote FernÁndeZ, Ignacio, EL derecho de autor en Internet, Granada, Comares, 2003, y Antequera PARILI, Ricardo, Estudios sobre derechos de autor y derechos afines, Madrid, Fundación Aisge, 2007.
} 
estadounidenses en diversas oportunidades han analizado la responsabilidad que corresponde a las empresas tecnológicas por las supuestas infracciones a los derechos de autor cometidas por los usuarios de sus servicios, particularmente en los casos de intercambios de obras protegidas a través de tecnologías como P2P. ${ }^{10}$ En el ámbito del copyrigth, esta responsabilidad se determina a través de la doctrina de la responsabilidad secundaria y la aplicación de los principios de safe harbor y el fair use.

\section{Supuestos de responsabilidad}

Bajo los principios del sistema jurídico estadounidense, provenientes en su mayor parte de la jurisprudencia, existen tres supuestos de responsabilidad del isP: la responsabilidad directa (direct copyright infringement), la responsabilidad contributiva (contributory copyright infringement) y la responsabilidad vicaria o delegada (vicarious copyright infringement). La primera corresponde a quien reproduce, distribuye o comunica una obra protegida sin la previa y expresa autorización de su autor o titular del derecho, quien es considerado el responsable directo de tal infracción. La contributiva se refiere al comportamiento de una persona que, con conocimiento, induce o contribuye a la producción de una infracción directa de los derechos de autor por parte de otra persona. La vicaria tiene lugar cuando existe la posibilidad de control o supervisión de una persona, que a su vez se beneficia económicamente de la infracción. ${ }^{11}$

Si bien es cierto que en la mayoría de los casos sólo se puede imputar infracción directa a los usuarios, el IsP también puede ser responsable cuando tiene conocimiento del alojamiento del material protegido. En 1993, mucho antes de la incorporación de los principios de la DMCA a la legislación de copyright, la Corte de Distrito de Florida, en Playboy Enterprises vs. George Frena,${ }^{12}$ determinó la responsabilidad de un proveedor de servicios de alojamiento como una infracción directa derivada del uso de unas de fotografías sin autorización

\footnotetext{
${ }^{10}$ Para un estudio amplio acerca del funcionamiento de estos sistemas y un análisis de la jurisprudencia europea y española en las redes P2P, véase LAstiRI SANTIAGo, MónICA, "La propiedad intelectual y las redes peer to peer. Estado actual", en Comercio electrónico: estructura operativa y jurídica, Buenos Aires, Argentina, Hammurabi, 2010, pp. 930-973.

"Véase De Freitas Straumann, Eduardo, "Experiencia en los Estados Unidos de América: Digital Millennium \& Copyright Act", XI Curso Académico Regional ompl/sGaE sobre Derecho de Autor y Derechos Conexos para Paises de América Latina: "El derecho de autor y los derechos conexos en el entorno digital", noviembre, 2005).

12 "Playboy Enterprises, Inc., Plaintiff, v. George Frena, d/b/a Techs Warehouse bBs Systems and Consulting, and Mark Dyess, 839 F. Supp. 1552 No. 93-489-Civ-J-20", Loundy. [Consulta: 25 de enero, 2017]. Disponible en http://www. loundy.com/CASES/Playboy_v_Frena.html
} 
de su autor. Consideró que dicho proveedor sabía que se estaban difundiendo.

Igualmente es importante citar la decisión de 21 de noviembre de 1995, de la Corte del Distrito Norte de California, también previa a la DMCA, en el caso Religious Technology Center vs. Netcom. ${ }^{13}$ En este caso el tribunal determinó que la legislación no impide la imposición de responsabilidad por infracción del derecho de autor sobre ciertas personas que no se han dedicado ellos mismos a la actividad infractora. La responsabilidad por la participación se establece cuando el demandado, con conocimiento de la actividad infractora, induce, causa o contribuye materialmente a la conducta infractora de otros.

\section{La doctrina de la responsabilidad secundaria}

La responsabilidad de los terceros, en este caso de las empresas tecnológicas en su función de ISP, siempre será secundaria (aunque pueda ser catalogada como una responsabilidad directa, como sucedió en el caso anteriormente citado), esto significa que para que proceda, necesariamente tiene que haber una responsabilidad primaria; en este caso, los responsables principales son los usuarios (tanto el que pone a disposición el archivo como el que lo descarga, independientemente del conocimiento de la infracción). ${ }^{14}$

En Estados Unidos existen dos doctrinas diferentes sobre la responsabilidad secundaria aplicables a los ISP por las vulneraciones que se producen en el ámbito del copyright conocidas como vicarious liability y contributory infringement.

La responsabilidad vicaria (vicarious liability) en general se refiere a la responsabilidad por las actuaciones de otra persona, tal como sucede con los empleadores por la actuación de sus empleados o de los padres o tutores por las personas a su cargo (responsabilidad por hecho ajeno). Este tipo de responsabilidad ha sido ampliamente desarrollada en la jurisprudencia estadounidense en relación con la protección del copyright.

En el caso de los ISP, es aplicable cuando el proveedor tiene la capacidad de controlar a los usuarios y obtiene un beneficio financiero al permitir la utilización de obras protegidas sin la autorización de sus autores. La DMCA establece las condiciones para eximir a la persona que suministra servicios de

\footnotetext{
13 "Religious Technology Center v. Netcom On-Line Com., 923 F. Supp. 1231 (N.D. Cal. 1995)", Justicia. [Consulta: 25 de febrero, 2017]. Disponible en http://law.justia.com/cases/federal/district-courts/FSupp/923/1231/1946287

${ }^{14}$ Tal como ha establecido la jurisprudencia, no se necesita la intención de infringir para determinar que hay infracción a los derechos de autor. Por lo tanto, un infractor inocente es culpable por la infracción. Decisión de la Corte de Distrito de Florida en el caso Playboy, citada por Antequera Parilli, Ricardo, Estudios sobre derechos de autor y derechos afines, Madrid, Fundación Aisge, 2007, p. 363.
} 
alojamiento a terceros, quien básicamente debe demostrar que no tenía conocimiento del contenido fraudulento y que la actividad no representa ningún beneficio económico para el ISP. La mayoría de los litigios relativos a la responsabilidad vicaria contra los intermediarios de internet están relacionados con acciones contra desarrolladores de software P2P y otras tecnologías que permiten el intercambio de archivos en formato digital.

La segunda doctrina se refiere a la institución denominada como contributory infringement (responsabilidad o infracción contributiva). Como su nombre lo indica, se produce cuando una parte, con conocimiento de la actividad infractora, induce, causa o materialmente contribuye a la conducta infractora de otra (siguiendo el texto de la sentencia del caso Religious Technology Center vs. Netcom). Esta situación es diferente a la anterior, ya que parte del conocimiento del ISP de un uso perjudicial a los derechos de los autor de los contenidos que se transmiten y exige una conducta activa que causa, induce o contribuye con la infracción. Esto explica por qué la sentencia mencionada se refiere a una responsabilidad directa.

La infracción contributiva también ha sido considerada por los tribunales estadounidenses en otros casos que involucran la actuación de los ISP. En la sentencia contra Napster, empresa pionera en popularizar las redes P2P y facilitar el intercambio de obras protegidas en formato digital, el IsP fue declarado culpable por contributory infringement. Se determinó que Napster había contribuido sustancialmente a la infracción proporcionando los servicios de apoyo que permitían a los usuarios encontrar y descargar música de manera ilegal. ${ }^{15}$.

\section{Los principios de safe harbor y fair use}

La principal innovación de la DMCA en el ámbito de los derechos de autor se concreta en la regulación de la responsabilidad de los ISP. La sección 512 se refiere a las limitaciones de responsabilidad relacionadas con material en línea y establece los principios del safe harbor como una exención a la responsabilidad de los ISP.

\footnotetext{
${ }^{15}$ La responsabilidad contributiva fue alegada en la demanda contra Napster. Si bien en este caso los demandantes admitieron que Napster no violaba en forma directa los derechos de autor de las obras que los usuarios compartian, si facilitaba la distribución del programa que contribuian a la violación. Sobre el caso y funcionamiento de Napster y otros sistemas P2P, véase GAMBOA, RAFAEL, "La nueva amenaza a la propiedad intelectual en la Red", en Derecho de Internet y Telecomunicaciones, Bogotá, Legis, 2003, pp. 495 y ss.
} 
Para establecer estas exenciones, la sección 512 diferencia entre cuatro clases de actividades de los ISP: $a$ ) la transmisión transitoria de comunicaciones; b) la memoria "caché"; c) el almacenamiento de información en sistemas o redes a discreción de los usuarios, y $d$ ) las referencias de enlaces que puedan conducir a los usuarios a material infractor.

En cuanto a la responsabilidad de los proveedores de alojamiento de contenidos suministrados por terceros, para que queden exentos por las violaciones al copyright, no deben tener conocimiento del contenido o la actividad relacionada con éste, ni ser conscientes de hechos o circunstancias que revelen una actividad infractora. En los casos en que el ISP tiene el derecho de controlar la mencionada actividad, tampoco deben percibir un beneficio económico derivado de tal conducta. Cuando el IsP tiene conocimiento de una actividad de este tipo, debe proceder a retirar el material infractor.

El tercer supuesto de safe harbor previsto en la DMCA se refiere a la información que reside en sistemas o redes a discreción de los usuarios. Por lo tanto, es aplicable a los servicios en la nube. Así lo ha determinado la jurisprudencia estadounidense. ${ }^{16}$ En líneas generales, el primer apartado c) de la sección 512 establece que los ISP no serán responsables por las actividades de los usuarios que consistan en almacenamiento de material que resida en un sistema o red controlada u operada por el proveedor de servicios, siempre que se cumplan las siguientes condiciones: $a$ ) que el ISP no tenga conocimiento de que el material alojado está infringiendo los derechos de autor, y que tan pronto lo tenga, elimine o inhabilite el acceso al material; $b$ ) que el ISP no reciba un beneficio financiero directamente atribuible a la actividad infractora; y c) que tan pronto como reciba la notificación sobre la infracción, responda rápidamente para remover o inhabilitar el acceso al material que se alega estar infringiendo. Esto es lo que se conoce en el Derecho estadounidense como notice and takedown (la acción de retiro del material después de una notificación).

El fair use se encuentra previsto en la sección 507 de la Copyrigth Act. ${ }^{17}$ De acuerdo con esta disposición, para que proceda esta excepción se deben considerar los siguientes factores: el propósito y el carácter del uso; la naturaleza

\footnotetext{
${ }^{16}$ Véase "Capitol Records, Inc. v. IIc, Exp. No. 07-9931 (S.D.N.Y. Agos 13, 2009)", Casetext. [Consulta: 20 de enero, 2017]. Disponible en https://casetext.com/case/capitol-records-inc-v-mp3tunes-3

${ }^{17}$ El fair use es una doctrina propia del derecho estadounidense que surgió de la interpretación jurisprudencial en este país como un límite al derecho exclusivo del autor a la reproducción de la obra. El fair use es vital en la protección del copyrigth en los Estados Unidos. Su importancia y su consideración en casos como los que nos ocupan es tal que el sitio web oficial del copyrigth del gobierno de este pais ofrece un índice con los principios y la aplicación para su uso. De esta manera, son más accesibles y comprensibles para el público. La base de datos incorpora diversas opiniones de los tribunales, incluyendo la categoría y el tipo de uso (música, contenidos en internet, digitalización, parodia).
} 
de la obra protegida; la cantidad y sustancia de la parte utilizada en relación con el todo; y el efecto del uso sobre el mercado potencial o el valor de la obra protegida. Estas cuatro defensas deben analizarse cuidadosamente para determinar si el uso del material protegido sin autorización es lícito de acuerdo con lo establecido en la ley. Al tratarse de una excepción, debe interpretarse de manera restrictiva y atendiendo a las circunstancias particulares de cada caso.

Diferentes tribunales han aplicado la doctrina fair use en relación con la determinación de la responsabilidad de los ISP. Si los usuarios usan material protegido bajo cualquiera de los factores que se consideran como un uso lícito, el ISP no puede asumir responsabilidad. En el caso que nos ocupa, la doctrina estadounidense se ha referido a esta excepción al indicar que el fair use funciona en la periferia de la aplicación del copyright en la nube. Lo anterior dado que es una excepción al acto principal de infracción y no una defensa separada contra las reclamaciones secundarias por infracción de responsabilidad. ${ }^{18}$

\section{El caso Capitol Records, Inc. v. MP3tunes}

De acuerdo con la DMCA, un ISP puede beneficiarse de los principios de safe harbor sólo cuando cumple con las condiciones establecidas en la sección 512 (i). En éstas resalta el deber de adoptar, implementar e informar a los suscriptores y titulares de la cuentas del sistema o red de una política que prevé la terminación de estas cuentas a quienes sean infractores reincidentes y únicamente cuando el ISP haya designado un agente para recibir las notificaciones sobre la infracción reclamada. Las notificaciones sobre la designación de estos agentes se hacen en la Copyright Office.

Para que el notice and take down proceda, la DMCA exige la identificación del material que es objeto de la actividad infractora y que debe ser removido o cuyo acceso debe ser inhabilitado. Cuando el IsP recibe una notificación de infracción, surge la obligación eliminar o deshabilitar el acceso a dicho material. ${ }^{19}$

En el caso Capitol Records, Inc. v. MP3tunes, la empresa tecnológica demandada ofrecía un servicio de nube híbrido (privado-público). A la vez, era propietaria de Sideload.com, un sitio de motor de búsqueda que permitía a los usuarios buscar enlaces en internet, descargar música y subirla a un archivo

\footnotetext{
${ }^{18}$ LeARY, Brian, "Safe Harbor Startups: Liability Rulemaking under the DMCA", New York University Law Review, núm. 87.

${ }^{19}$ Esta obligación ofrece a los titulares de derechos de autor un recurso adecuado para casos individuales de infracción. No obstante, debido a que los titulares de los derechos tienen la carga de supervisar la actividad de los usuarios por infracción, los avisos de eliminación sólo son eficaces cuando la actividad del usuario es pública. LEARY, BRIAN, "Safe Harbor Startups: Liability Rulemaking under the DMCA", New York University Law Review, núm. 87.
} 
digital de MP3tunes. Una vez que una canción se incorpora al archivo en línea, se puede descargar desde el dispositivo de cualquier usuario, lo cual permite utilizar y compartir el material protegido sin el consentimiento del autor.

Siguiendo las previsiones de la DMCA, MP3tunes registró un agente en la oficina del copyright para recibir las notificaciones de las supuestas infracciones a los propietarios de estos derechos y proporcionó la información de contacto de este agente en ambos sitios. En septiembre de 2007, MP3tunes recibió una notificación de EMI, donde se identificaban 350 títulos de canciones y enlaces indexados en Sideload.com que conectaban a los usuarios con sitios que infringían los derechos de autor de EMI. MP3tunes eliminó los enlaces identificados por EMI y cerró las cuentas de los infractores reincidentes, pero no removió las copias de las canciones de estos archivos.

La demandante exigió responsabilidad secundaria vicaria o contributiva ante el Tribunal de Distrito de los Estados Unidos para el distrito sur de Nueva York $^{20}$ por infracción al copyright a MP3tunes. Alegó que la empresa proporcionaba los medios que permitían a los usuarios finales violar el copyright; argumentó además que la empresa no era elegible para protección de la DMCA porque no había implementado una política razonable de infracción, pues no permitía la identificación de los usuarios que habían utilizado las obras protegidas indicadas en la notificación de eliminación. Asimismo, no eliminó de manera expedita las canciones de los archivos de los usuarios que fueron transferidos desde los sitios web identificados en la notificación correspondiente.

La corte determinó que MP3tunes si había cumplido con las previsiones de la DMCA (en cuanto al registro del agente y el sistema del notice and take down) y por lo tanto era elegible para las protecciones de la DMCA. Sin embargo, también dictaminó que la empresa no sólo tenía el deber de eliminar los enlaces a los materiales infractores publicados en Sideload.com, sino además la obligación de eliminar las canciones almacenadas en los archivos personales de los usuarios que se descargaron de dichos enlaces. Por esta razón, la corte atribuyó a MP3Tunes responsabilidad por contributory infringement.

\footnotetext{
${ }^{20}$ Debido a la naturaleza de los servicios y a la facilidad de reproducción de la obra, las violaciones al copyright en el ámbito del cloud computing pueden producir efectos fuera del país donde se encuentra el Isp. En este caso, la acción se ejerció ante esta jurisdicción por ser la competente para conocer la demanda al involucrar dos empresas estadounidenses y estar relacionada directamente con protección al copyright prevista en la DMCA. Es importante hacer esta aclaratoria debido a que en los conflictos derivados de la prestación de servicios en la nube puede presentarse un problema para determinar la jurisdicción competente ante la posibilidad de involucrar distintas legislaciones que se consideren aplicables a los hechos. Sobre esta problemática, véase Del Poyo, Rafael, "Cloud Computing: aspectos jurídicos clave para la contratación de estos servicios", Revista Española de Relaciones Internacionales, núm. 4. [Consulta: 28 de agosto, 2017]. Disponible en http://reri.difusionjuridica.es/index.php/RERI/article/view/45/43
} 
En la referida sentencia, puede observarse que, aunque la empresa demandada había cumplido con uno de los requisitos previstos para la exoneración de responsabilidad (la política del notice and takedown) incumplió su obligación al no remover las canciones y el material fonográfico que infringía el copyright con posterioridad a la notificación de esta situación. Estos fueron los hechos que motivaron la decisión la Corte al atribuir la responsabilidad contributiva. Ésta, como se recordará, procede cuando existe conocimiento de la empresa tecnológica que facilita los medios para la infracción directa de los derechos de autor por parte de los usuarios, el cual quedó evidenciado con la notificación recibida y la acción de la demandada al eliminar los enlaces en Sideload.com que dirigían al material protegido.

Es importante resaltar que, en cuanto a la actividad de los usuarios, el tribunal distingue entre los infractores flagrantes, que saben que carecen de autorización, y los usuarios que descargan contenido para su entretenimiento personal y lo usan para fines permitidos por la ley (fair use). Se consideran infractores flagrantes aquellos que cargan contenido y permiten conscientemente que otros lo copien. La distinción que realiza el juzgador es importante en relación con la doctrina del fair use, ya que ésta puede proteger a un usuario que copia una canción usando los servicios de la nube (la grabación personal o copia privada en el caso Sony), pero el fair use no excusa a quien usa estos servicios para compartir y distribuir el material protegido. ${ }^{21}$ Esta sentencia afirma nuevamente la protección de las empresas que suministran una determinada tecnología y la aplicación a los proveedores de servicios en la nube de los principios de la DMCA. A pesar de los avances que esto puede representar en cuanto a las medidas de protección para los titulares del copyright, se debe tener en cuenta que cuando los servicios en la nube son privados es difícil detectar una conducta infractora. Ello pone de manifiesto la ineficacia de los avisos de eliminación en estos casos.

Recordemos que para que el notice and take down proceda, la DMCA exige la identificación del material que es objeto de la actividad infractora. Al tratarse de un ámbito privado, las empresas que suministran este tipo de servicios rara vez recibirán notificaciones de eliminación. Esta circunstancia es mencionada por la doctrina anglosajona, que advierte que la decisión sobre MP3tunes no se extiende al intercambio de obras protegidas en nubes privadas sin un servicio como Sideload porque el take and down notice no tendría ningún efecto. ${ }^{22}$

\footnotetext{
${ }^{21}$ Leary, Brian, "Safe Harbor Startups: Liability Rulemaking under the DMCA", New York University Law Review, núm. 87.

22 LeARY, BRIAN, "Safe Harbor Startups: Liability Rulemaking under the DMCA", New York University Law Review, núm. 87.
} 
A pesar de estas consideraciones, es importante recordar que tradicionalmente la copia de obras protegidas para su uso privado también constituye una excepción a los derechos exclusivos de explotación de los autores. Sin embargo, si bien la copia privada en un principio no perturbaba los derechos de los autores, esta situación ha cambiado con el desarrollo de la tecnología y la posibilidad de transferencias de grandes cantidades de información en los intercambios privados de archivos que permiten la reproducción íntegra de la obra. ¿En qué medida las normas jurídicas o la jurisprudencia pueden sentar las bases para evitar esta situación en los servicios de nubes privadas? Con esta interrogante finalizamos nuestro estudio, y dejamos al lector un nuevo campo para la investigación sobre las vulneraciones a la propiedad intelectual en este ámbito.

\section{Conclusiones}

Luego del análisis del caso descrito y de las normas y la jurisprudencia citadas en la parte principal de este trabajo podemos extraer las siguientes conclusiones.

El intercambio de archivos digitales en la nube no es una tecnología ilegal, como tampoco lo son los diversos sistemas que permiten el intercambio de obras digitales protegidas por el copyright en internet. La doctrina Sony es aplicable a estos casos, ya que la tecnología no sólo permite usos ilícitos, sino que también puede utilizarse ampliamente con fines legítimos significativos y bajo el amparo de la normativa jurídica que protege el copyright.

En los Estados Unidos el safe harbor y el fair use son doctrinas esenciales en la legislación sobre copyright y han sido tomadas en cuenta en numerosos casos en los que se discute la responsabilidad de los ISP por la transmisión de obras digitales sin la correspondiente autorización de los autores. Aunque el tercer supuesto de safe harbor es aplicable a los servicios de cloud computing, para poder beneficiarse de la exención de responsabilidad, el ISP debe implementar la política del notice and takedown y cumplir con la obligación de retirar el material o bloquear el acceso una vez que tiene conocimiento de la actividad infractora. Si el proveedor de servicios cumple con estos requisitos, califica para la protección y es inmune a los daños y perjuicios causados por los usuarios.

Los usuarios pueden acceder y utilizar material protegido por el copyright sin el consentimiento del autor bajo principios de fair use. Esta doctrina puede proteger a un usuario que copia o graba una obra para su uso personal, por medio de los servicios que ofrece la nube, y está reconocida en el ToDA. Sin embargo, es importante tener presente que el fair use no protege a los usuarios que utilizan los servicios de la nube para compartir el material protegido bajo el copyright. 
Por lo tanto, el análisis del caso concreto es fundamental a la hora de aplicar esta doctrina.

En casos como el analizado en el presente trabajo, la responsabilidad de las empresas tecnológicas siempre ha de establecerse bajo la doctrina de la responsabilidad secundaria (vicarious liability o contributory infringement). Para que proceda, necesariamente tiene que haber una responsabilidad primaria derivada de la conducta de los usuarios (tanto del que pone a disposición el archivo como del que lo descarga, independientemente del conocimiento de la infracción).

Respecto a la adecuación de la DMCA para resolver los conflictos entre el copyright y el uso de los servicios del cloud computing, se observa que en el caso Capitol Records vs. MP3tunes, a pesar de las pretensiones de la parte demandante, el tribunal determinó que un proveedor de servicios en la nube sí puede beneficiarse de la protección que otorga la DMCA, siempre que cumpla los requisitos para beneficiarse de esta exención. MP3tunes efectivamente había cumplido al poner en práctica una política eficaz de notice and takedown, pero falló en retirar el material almacenado en los archivos privados de los usuarios, de ahí que fuera condenado por responsabilidad contributiva.

La naturaleza de determinados servicios, como las nubes privadas, representa un nuevo reto en la protección jurídica de los derechos de autor en este ámbito ya que puede incrementar la masificación en el uso de copias privadas. Sin embargo, no debe olvidarse que la copia privada también constituye una excepción al copyright, que permite el uso privado sin el consentimiento del autor. También debemos tener presentes las conclusiones del caso Sony respeto a la aplicación del fair use en la grabación personal. No obstante, la determinación de la infracción dependería de los hechos concretos y la problemática en este tipo de servicio se deriva precisamente de la dificultad de detectar estos hechos y valorar si el uso privado corresponde a una de las excepciones permitidas. 


\section{Bibliografía}

Antequera Parilli, Ricardo, Estudios sobre derechos de autor y derechos afines, Madrid, Fundación Aisge, 2007.

Aristizabal Velásquez, DaVid, "Luces y sombras de las nuevas tendencias de la regulación de contenidos informáticos en los Estados Unidos de Norteamérica”, Revista Ces Derecho, vol. 3, núm. 1.

“Capitol Records, Inc. v. LLc, Exp. No. 07-9931 (S.D.N.Y. Agos 13, 2009)”, Casetext. [Consulta: 20 de enero, 2017]. Disponible en https://casetext.com/case/capitol-records-inc-v-mp3tunes-3

Carbajo Cascón, Fernando, "La propiedad intelectual como objeto del comercio electrónico", en María Jesús Moro Almaraz, Autores, consumidores y comercio electrónico, Madrid, Colex, 2004.

De Freitas Straumann, Eduardo, "Experiencia en los Estados Unidos de América: Digital Millennium \&t Copyright Act”, XI Curso Académico Regional omPI/sGAE sobre Derecho de Autor y Derechos Conexos para Países de América Latina: "El derecho de autor y los derechos conexos en el entorno digital”, noviembre, 2005.

Del Poyo, Rafael, "Cloud Computing: aspectos jurídicos clave para la contratación de estos servicios", Revista Española de Relaciones Internacionales, núm. 4. [Consulta: 28 de agosto, 2017]. Disponible en http://reri.difusionjuridica.es/ index.php/RERI/article/view/45/43

Gamboa, Rafael, "La nueva amenaza a la propiedad intelectual en la Red", en Derecho de Internet y Telecomunicaciones, Bogotá, Legis, 2003.

Garrote Fernández, Ignacio, EL derecho de autor en Internet, Granada, Comares, 2003.

LASTIRI SANTIAgo, Mónica, "La propiedad intelectual y las redes peer to peer. Estado actual”, en Comercio electrónico: estructura operativa y jurídica, Buenos Aires, Argentina, Hammurabi, 2010.

Leary, Brian, "Safe Harbor Startups: Liability Rulemaking under the DMCA", New York University Law Review, núm. 87.

"Playboy Enterprises, Inc., Plaintiff, v. George Frena, d/b/a Techs Warehouse вBS Systems and Consulting, and Mark Dyess, 839 F. Supp. 1552 No. 93-489-Civ-J-20", Loundy. [Consulta: 25 de enero, 2017]. Disponible en http://www.loundy.com/CASES/Playboy_v_Frena.html

"Religious Technology Center v. Netcom On-Line Com., 923 F. Supp. 1231 (N.D. Cal. 1995)”, Justicia. [Consulta: 25 de febrero, 2017]. Disponible en http://law. justia.com/cases/federal/district-courts/FSupp/923/1231/1946287 